\title{
To Assess Predictive Value of Cord Blood Bilirubin and Albumin for Significant Neonatal Hyperbilirubinemia: A Prospective Study from India
}

\begin{abstract}
Aim: To assess the usefulness of the cord blood bilirubin and cord albumin estimation as a predictor of subsequent neonatal hyperbilirubinemia in a healthy term infants and to compare their predictive abilities: a prospective study.
\end{abstract}

Material and Method: This study enrolled 200 full term neonates during the study period. Bilirubin and albumin estimation was done from cord blood, 1st day, 3rd day and 5th day bilirubin estimation was done from peripheral venous blood. Relationship was seen between level of albumin and bilirubin.

Result: Cord blood bilirubin level of $>2 \mathrm{mg} / \mathrm{dl}$ had a sensitivity of $90 \%$ and specificity of $53.89 \%$, positive predictive value $17.8 \%$ and negative predictive value of $98 \%$ in predicting the risk of neonatal hyperbilirubinemia. ( $\mathrm{p}<.001)$. Cord blood albumin level of $\leq 2.6 \mathrm{gm} / \mathrm{dl}$ had a sensitivity of $80 \%$ and specificity of $86.67 \%$, positive predictive value $40 \%$ and negative predictive value of $97.5 \%$ in predicting the risk of neonatal hyperbilirubinemia ( $p<.0001$ ) 1st day bilirubin level of $\geq 5.7 \mathrm{mg} / \mathrm{dl}$ had a sensitivity of $90 \%$ and specificity of $82.22 \%$, positive predictive value of $36 \%$ and negative predictive value of $98.7 \%$ in predicting the risk of neonatal hyperbilirubinemia( $p<.0001)$.

Conclusion: Cord bilirubin and albumin can be used as predictor for hyperbilirubinemia and for early review for jaundice especially in developing countries where regular follow up is difficult.

Keywords: Cord Bilirubin; Cord Albumin; Neonatal Hyperbilirubinemia; Prediction risk

\section{Introduction}

Neonatal hyperbilirubinemia needs appropriate and timely treatment no matter whether it may arise from physiological or pathological causes [1]. However left untreated physiological neonatal jaundice may tend to resolve spontaneously with increasing maturity of liver in most of neonates, whereas in pathological jaundice in substantial number of cases especially those with hemolytic states such as Rh, ABO incompatibility, minor blood group incompatibility [2,3] or G6PD deficiency, the hyperbilirubinemia may reaches to a level which is toxic enough to cause brain damage [4]. Bilirubin enters the brain as free (unbound) bilirubin or as bilirubin bound to albumin in the presence of a disrupted blood-brain barrier in conditions like severe metabolic acidosis, asphyxia and prematurity and damaged the brain neurons primarily and then also causes damage to astrocytes, oligodendrocytes and microglia [5]. Bilirubin induced neuronal damage is most commonly seen in the basal ganglia, various cranial nerve nuclei, other brainstem nuclei, cerebellar nuclei, hippocampus, and anterior horn cells of the spinal cord. Microscopically, there is necrosis, neuronal loss, and gliosis [6]. The neurological complication caused by hyperbilirubinemia includes bilirubin induced neurological dysfunction, acute bilirubin induced encephalopathy and chronic bilirubin encephalopathy (kernicterus). Acute bilirubin encephalopathy is characterized by three different phases with early phase characterized by hypotonia, lethargy, high-pitched cry, and poor suck. The intermediate phase is characterized by Hypertonia of extensor muscles (with opisthotonus, rigidity, oculogyric crisis, and retrocollis), irritability, fever, and seizures. The advanced phase have features of pronounced opisthotonus (although hypotonia replaces hypertonia after approximately 1 week of age), shrill cry, apnea, seizures, coma, and death $[7,8]$.

Chronic bilirubin encephalopathy (kernicterus) is featured by athetosis, complete or partial sensorineural deafness (auditory neuropathy), limitation of upward gaze, dental dysplasia, and sometimes, intellectual deficits [9]. Early discharge of healthy term newborns after normal vaginal delivery has become a common practice, because of medical reasons like prevention of nosocomial infections, social reasons like in early naming ceremony as practiced in Muslim community, and also due to economical constrains. Financial constraints, family and medical consideration has led to early discharge of the healthy term neonates after delivery. Thus the recognition, follow up and early treatment of Jaundice has become more difficult as a result of earlier discharge from the hospital $[10,11]$.

American Academic of Pediatrics recommends that newborn discharged within 48 hours should have a follow-up visit after 48 to 72 hours for any significant jaundice and other problems [12]. This recommendation is not appropriate for our country due to limited follow-up facilities in the community. These babies may develop jaundice which may be over looked or delay in recognition unless the baby is closely monitored 
on regular follow-up. The concept of prediction of Jaundice offers an attractive option to pick up babies at risk of neonatal hyperbilirubinemia. Since the physical examination is not a reliable measure of the estimation of serum bilirubin. Under these circumstances it would be desirable to be able to predict the risk of significant jaundice, in order to implement early treatment and thereby minimize the risk of bilirubin dependent brain damage [13].

By predicting the newborns developing significant neonatal jaundice early at birth, we can design and implement the follow-up in the high risk groups effectively. Neonatal hyperbilirubinemia is a cause of concern for the parents as well as the pediatricians. Early discharge of healthy term newborns after delivery has become a common practice because of medical and social reasons and economic constraints. It is significant that most common cause for readmission during the early neonatal period is Hyperbilirubinemia [14]. The present study was therefore conducted to find out the critical value of cord blood bilirubin and cord blood albumin in predicting the subsequent development of significant neonatal jaundice requiring interventions like phototherapy or exchange transfusion which otherwise would have missed due to the practices of early discharge and with the limited follow-up facilities in the community.

\section{Materials and Methods}

This was a hospital based, prospective study and was conducted in Department of Pediatrics, Umaid hospital attached to Dr. S. N. Medical College, Jodhpur. The study group consisted of 200 full term healthy neonates delivered at Umaid Hospital from December 1st 2012 to November 30th 2013. These neonates were followed from birth to 5 th postnatal day. Ethical clearance was obtained from the institutional research board (IRB) of Dr. S. N. medical college, Jodhpur.

Informed written parental consent was obtained from all cases. Data was collected as per the performa. Questionnaire method, maternal case file, and examination of the newborn were used to obtain the required data. Babies were examined daily and looked for evidence of jaundice, sepsis, illness or birth trauma. Weight of the newborn was recorded and gestational age calculated. All the babies were followed up daily for first 5 postnatal days because peak serum bilirubin occurs between 3rd and 5th day. Cord blood was collected at birth for estimation of bilirubin and albumin. First day serum bilirubin was estimated using blood drawn 24 hours after birth. Blood was also drawn on 3rd and 5th day. Peripheral venous blood was used to measure serum bilirubin.

The main outcome of the study was inferred in terms of hyperbilirubinemia. Serum bilirubin $\geq 17 \mathrm{mg} / \mathrm{dl}$ after 72 hours of life was taken as Hyperbilirubinemia needing phototherapy and treatment is advised to all those full term healthy babies with serum bilirubin level of $\geq 17 \mathrm{mg} / \mathrm{dl}$ after 72 hours of life, as per the American academy of Pediatrics practice parameter, 2004 [12]. All the data was entered in Microsoft excel sheet and SPSS version 16 for window was used. Statistical data were analyzed with the independent sample t test and the descriptive analysis, chi-square tests and ANOVA. Sensitivity, specificity, negative and positive predictive value of the test was calculated. The critical cord bilirubin, cord albumin and first day bilirubin levels having the highest sensitivity and specificity were determined with the Receiver operating characteristics (ROC) curve analysis. Cord serum bilirubin, cord serum albumin and first day serum bilirubin concentration were used for developing 'prediction test'. The sensitivity and specificity were calculated for predicting Hyperbilirubinemia.

\section{Results}

In the present study a total of 200 babies were registered. Out of these $55 \%$ were male and $45 \%$ were female. The male to female ratio was 1.22: 1 . There was no significant difference in the number of male and female babies. Out of 110 male neonates, $12(10.9 \%)$ newborns developed significant hyperbilirubinemia while out of 90 female neonates, 8 (8.89\%) newborns developed significant hyperbilirubinemia. This difference was not found significant statistically ( $\mathrm{p}>0.05$ ).

The majority of the cases that is $101(50.5 \%)$ babies in this study belonged to birth weight group of $2.5-2.8 \mathrm{Kg}$ while 72 cases (36\%) were from 2.9-3.2 Kg group and 27 cases (13.5\%) has birth weight of $>3.2 \mathrm{Kg}$. Out of 101 babies whose birth weight group was 2.5-2.8 Kg 10 (9.90\%) babies developed significant Neonatal Hyperbilirubinemia, in the birth weight group of 2.9$3.2 \mathrm{Kg}$ whereas $8(11.11 \%)$ babies out of 72 babies and in the birth weight group of $>3.2 \mathrm{Kg}$ only $2(7.40 \%)$ babies out of 27 babies has developed significant Neonatal Hyperbilirubinemia. In the present study there was no significant association between the birth weight of the newborn babies and the development of significant Neonatal Hyperbilirubinemia $(\mathrm{p}>0.9)$.

Out of total 200 babies, mothers of $30(15 \%)$ babies received oxytocin for the induction of labour and mothers of remaining $170(85 \%)$ babies did not receive oxytocin. Among 30 babies whose mothers received oxytocin $6(20 \%)$ babies developed significant neonatal hyperbilirubinemia while out of 170 babies whose mothers did not receive oxytocin 14 (8.24\%) babies developed significant neonatal hyperbilirubinemia. In the present study there was significant association between the babies whose mother was given oxytocin for induction of labour and significant neonatal hyperbilirubinemia $(\mathrm{p}<.05)$.

Sex, religion, geographical area, birth weight, parity of mother, maternal gestational hypertension, mode of delivery, time of initiation of breast feeding is not associated with significant neonatal hyperbilirubinemia. In the present study mean value of the cord serum bilirubin was significantly higher in the babies who developed significant neonatal hyperbilirubinemia later $(2.72 \mathrm{mg} / \mathrm{dl})$ than the babies who did not develop neonatal hyperbilirubinemia $(1.99 \mathrm{mg} / \mathrm{dl})(\mathrm{p}<.001)$

Serum bilirubin level profile in first 5 postnatal days also infers that serum bilirubin level was significantly higher in the babies who developed significant neonatal hyperbilirubinemia later compared to the babies who did not develop neonatal hyperbilirubinemia $(p<.001)$. For the prediction of significant neonatal hyperbilirubinemia, the cut off value of cord serum bilirubin of $>2 \mathrm{mg} / \mathrm{dl}$ was chosen on the basis of receiver operating characteristics (ROC) curve analysis. In the present study the cord serum bilirubin $>2 \mathrm{mg} / \mathrm{dl}$ having the sensitivity $90 \%$, specificity $53.89 \%$, positive predictive value $17.8 \%$ and negative predictive value $98 \%$ in the prediction of the neonatal hyperbilirubinemia ( $p<.001$ ) (Figure 1). 


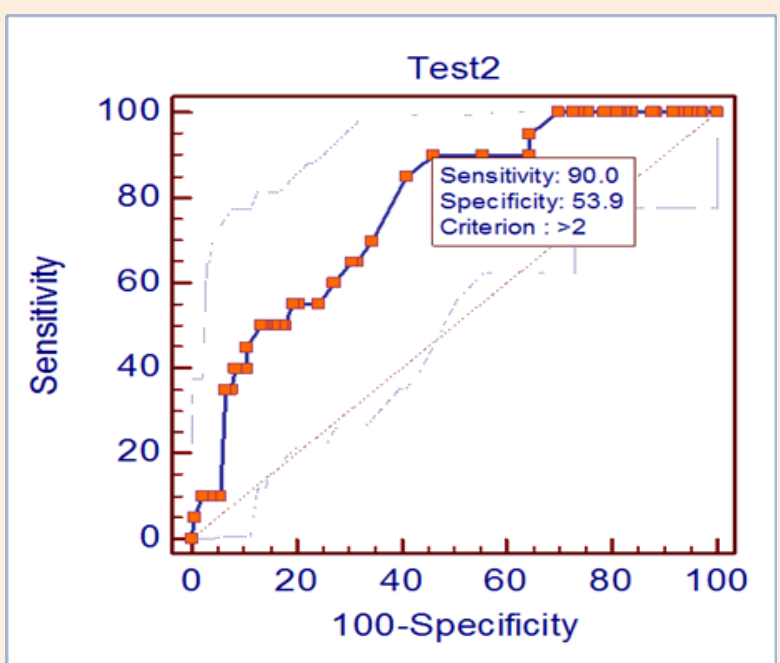

Figure 1:Association between the critical cord bilirubin level and the neonatal hyperbilirubinemia.

For the prediction of significant neonatal hyperbilirubinemia, the cut off value of cord serum albumin of $\leq 2.6 \mathrm{gm} / \mathrm{dl}$ was chosen, on the basis of receiver operating characteristics (ROC) curve analysis. In the present study the cord serum albumin $\leq 2.6$ gm/dl having the sensitivity $80 \%$, specificity $86.67 \%$, positive predictive value $40 \%$ and negative predictive value $97.5 \%$ in the prediction of the neonatal hyperbilirubinemia $(p<.0001)$ (Figure 2).

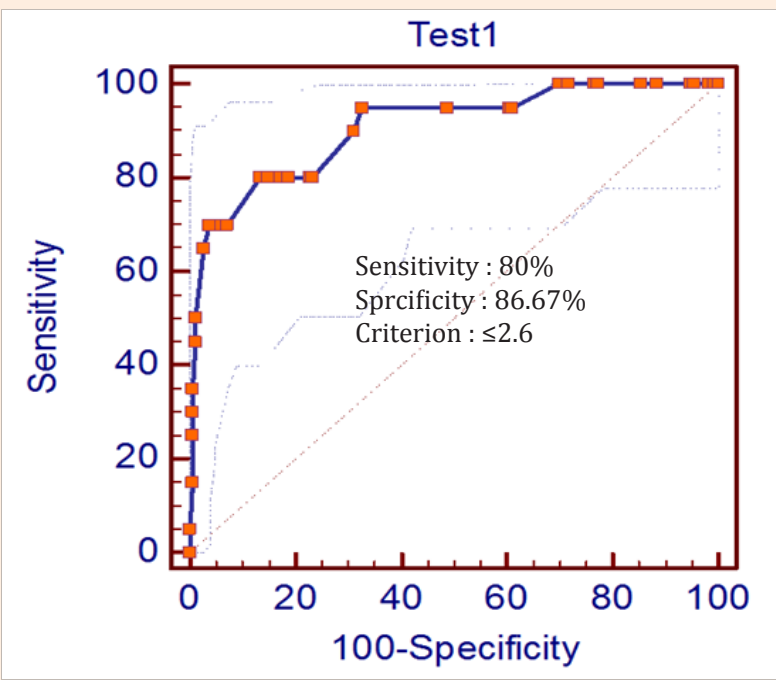

Figure 2: Association between the critical cord albumin level and the neonatal hyperbilirubinaemia.

For the prediction of significant neonatal hyperbilirubinemia, the cut off value of first day serum bilirubin of $>5.7 \mathrm{mg} / \mathrm{dl}$ was chosen, on the basis of receiver operating characteristics (ROC) curve analysis .1st day bilirubin level of $\geq 5.7 \mathrm{mg} / \mathrm{dl}$ had a sensitivity of $90 \%$ and specificity of $82.22 \%$, positive predictive value of $36 \%$ and negative predictive value of $98.7 \%$ in predicting the risk of neonatal hyperbilirubinemia. $(\mathrm{p}<.0001)$ (Figure 3).

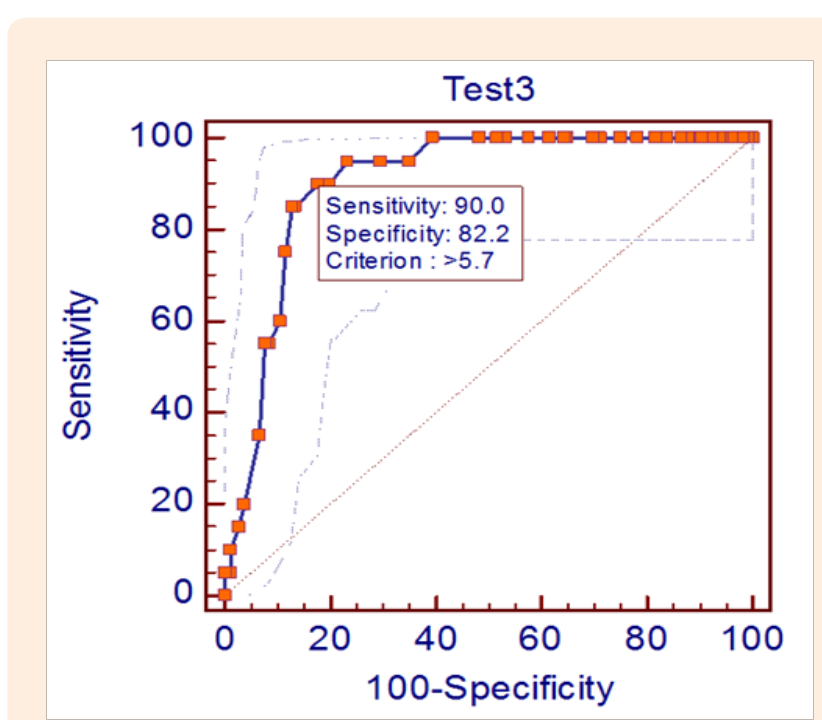

Figure 3: Association between the first day bilirubin level and the neonatal hyperbilirubinemia.

\section{Discussion}

In this present study, we assessed the ability of cord bilirubin, cord albumin and first day bilirubin level to be a tool for screening for the risk of subsequent neonatal jaundice. In the present study, study group was uniformly distributed with 110 male and 90 female babies and there was no significant correlation $(p=0.059)$ between the development of significant neonatal hyperbilirubinemia and the sex of the newborn. The present study is in correlation with the study done by Taksande et al. [13] and Rostami and Mehrabi [15]. However, Maisels and Kring [16] and Satrya et al. [17] showed that male sex has more risk of readmission for neonatal hyperbilirubinemia, this could be explained on the basis that in developing countries male neonates are taken care more in comparison to the female neonate because of gender discrimination prevalent in society of the developing countries and the same was seen in our study as our study enrolled neonates whose parents were from rural background.

In our study religion, geographical area, birth weight, parity of mother, maternal gestational hypertension, mode of delivery and time of initiation of breast feeding is not associated with significant neonatal hyperbilirubinaemia. Sun G et al. [18] and Sahu et al. [19] showed that there is no correlation between the neonatal hyperbilirubinemia and the birth weight of the newborn. ( $p>0.05)$. Sun $\mathrm{G}$ et al. [18], in a study showed that there is no correlation between the neonatal hyperbilirubinemia and the gestational age of the newborn ( $p>0.05)$. Taksande et al. [13], Rostami and Mehrabi [15], Satrya et al. [17], Knudsen [20] and Awasthi and Rehman [21] showed that there is no significant association between the mode of delivery and neonatal hyperbilirubinemia as seen in our study. This could be explained as the metabolism of bilirubin does not depend upon the mode of delivery. Awasthi and Rehman [21], showed there is no significant correlation between the timing of initiation of breast feeding and neonatal hyperbilirubinemia. Taksande et al. [13] 
and Awasthi and Rehman [21] showed no correlation between the neonatal hyperbilirubinemia and maternal gestational hypertension because maternal hypertension will not have any effect on the metabolism of bilirubin.

In the present study, there was significant association ( $p$ $<0.05$ ) between the babies given fluids and other medications with the development of neonatal hyperbilirubinemia. But there is a significant association between the induction of labour with oxytocin and the neonatal hyperbilirubinemia ( $p<0.05)$ The present study is in correlation with Taksande et al. [13], Rostami and Mehrabi [15], Awasthi and Rehman [21] and Oral E et al. [22]. This could be explained by the mechanism of hyperbilirubinemia with Oxytocin induction of labour- Oxytocin induces hyponatremia and hypo-osmolality in the mother by virtue of its anti-diuretic and saluretic effects. These biochemical changes are aggravated by the infusion of electrolyte-free dextrose solution used as a vehicle for administration of oxytocin Transplacentally transmitted hypo-osmolality in the fetal blood, leads to enhanced osmotic fragility of the red blood cells. The swollen and hyper fragile erythrocytes are easily trapped by the spleen resulting in net higher bilirubin production [22].

In the present study, on ROC curve analysis critical cord bilirubin level $>2 \mathrm{mg} / \mathrm{dl}$ with high sensitivity and high specificity is selected. The probability that a neonate with cord bilirubin $>2 \mathrm{mg} / \mathrm{dl}$ would later become hyperbilirubinaemia (positive predictive value) was $17.8 \%$. The negative predictive value, the probability of non-hyperbilirubinemia given a cord bilirubin $\leq 2 \mathrm{mg} / \mathrm{dl}$ was $98 \%$. If a child become hyperbilirubinemic, the probability that the cord bilirubin was $>2 \mathrm{mg} / \mathrm{dl}$ was $90 \%$ (sensitivity). Given a non-hyperbilirubinemic child, the probability that the cord bilirubin was $\leq 2 \mathrm{mg} / \mathrm{dl}$ was $53.9 \%$ (specificity).

Several studies are published on the usefulness of cord bilirubin concentration in prediction of hyperbilirubinemia. Taksande et al. [13], showed that the cord bilirubin level $>2 \mathrm{mg} / \mathrm{dl}$ has a sensitivity $89.5 \%$, specificity $85 \%$, negative predictive value of $98.7 \%$ and positive predictive value of $38.8 \%$ in correlation with the present study. Knudsen et al. [20], established that if the cord bilirubin was below $20 \mu \mathrm{mol} / \mathrm{l}, 2.9 \%$ became jaundiced, as opposed to $85 \%$ if the cord bilirubin was above $40 \mu \mathrm{mol} / \mathrm{l}$. Furthermore, $57 \%$ of jaundiced infants with cord bilirubin above $40 \mu \mathrm{mol} / \mathrm{l}$ required phototherapy, but only $9 \%$ if the cord bilirubin was $40 \mu \mathrm{mol} / \mathrm{l}$ or lower $(0.008)$ in correlation with the present study. Nahar et al. [23] showed that the cord bilirubin level $\geq 2.5 \mathrm{mg} / \mathrm{dl}$ has a sensitivity $77 \%$, specificity $98.6 \%$, with negative predictive value of $96 \%$ in correlation with the present study. Bernaldo and Segre [24] in 2005 showed that the cut off point for unconjugated bilirubin in cord blood was $\geq 2.0 \mathrm{mg} / \mathrm{dl}$ the probability that the newborn would need phototherapy was $53 \%$. When cord blood bilirubin was $2.5 \mathrm{mg} / \mathrm{dl}$ the probability needing phototherapy was $72 \%$, when the level was $3.0 \mathrm{mg} / \mathrm{dl}$, the probability of needing treatment was $86 \%$, and if it was $3.5 \mathrm{mg} / \mathrm{dl}$, the probability went up to $93 \%$. This could be explained because the newborns with higher cord bilirubin level have more rapid increase in serum bilirubin and have high chances of neonatal hyperbilirubinemia and more chances of requiring phototherapy.

Satrya et al. [17] and Sun et al. [18] studies are in correlation with the present study. Rostami and Mehrabi [15] on their study to identify healthy newborns at risk for developing significant hyperbilirubinemia by measuring bilirubin level in cord blood in 643 full term infants. Serum bilirubin level was obtained on umbilical cord serum and on day three of age. The total bilirubin $\geq 239 \mu \mathrm{mo} 1 / 1(14 \mathrm{mg} / \mathrm{dl})$ was defined as significant hyperbilirubinemia. They concluded that cord serum bilirubin level cannot identify newborns with subsequent significant hyperbilirubinemia. Our study infers that cord serum bilirubin $>2 \mathrm{mg} / \mathrm{dl}$ can be used as an early predictor of neonatal hyperbilirubinemia. This difference could be because of geographical difference in the study population.

In the present study, on ROC curve analysis critical cord albumin level $\leq 2.6 \mathrm{gm} / \mathrm{dl}$ with high sensitivity and high specificity is selected. The probability that a neonate with cord albumin $\leq 2.6 \mathrm{gm} / \mathrm{dl}$ would later become hyperbilirubinaemia (positive predictive value) was $40 \%$. The negative predictive value, the probability of non-hyperbilirubinemia given a cord albumin $>2.6 \mathrm{gm} / \mathrm{dl}$ was $97.5 \%$. If a child becomes hyperbilirubinemic, the probability that the cord albumin was $\leq 2.6 \mathrm{gm} / \mathrm{dl}$ was $80 \%$ (sensitivity). Given a non-hyperbilirubinemic child, the probability that the cord albumin was $>2.6 \mathrm{gm} / \mathrm{dl}$ was $86.67 \%$ (specificity).

A study done by Suchanda Sahu et al. [19], showed the prediction of significant hyperbilirubinemia by measuring cord blood albumin. $82 \%$ of neonate who had cord blood albumin level $<2.8 \mathrm{~g} / \mathrm{dl}$ developed hyperbilirubinemia. The present study infers that cord serum albumin $\leq 2.6 \mathrm{gm} / \mathrm{dl}$ can be used as an early predictor of neonatal hyperbilirubinemia. Cord serum bilirubin being more sensitive than cord serum albumin, is more effective to pick the babies who develop significant neonatal hyperbilirubinaemia when comparing each other.

In the present study, on ROC curve analysis critical 1stday bilirubin level with high sensitivity and high specificity $>5.7 \mathrm{mg} /$ $\mathrm{dl}$ is selected. The probability that a neonate 1st day bilirubin higher than $>5.7 \mathrm{mg} / \mathrm{dl}$ would later become hyperbilirubinemia (positive predictive value) was $36 \%$. The negative predictive value, the probability of non-hyperbilirubinemia given a 1st day bilirubin $\leq 5.7 \mathrm{mg} / \mathrm{dl}$ was $98.7 \%$. If a child become hyperbilirubinemic, the probability that the 1st day bilirubin was $>5.7 \mathrm{mg} / \mathrm{dl}$ was $90 \%$ (sensitivity). Given a nonhyperbilirubinemic child, the probability that the 1st day bilirubin was $\leq 5.7 \mathrm{mg} / \mathrm{dl}$ was $82.2 \%$ (specificity).

Randev and Grover [25], in a study, a total of 200 neonates were enrolled, 24 neonates (i.e., 12\%) developed hyperbilirubinemia. The mean first day TSB value in the neonates who subsequently developed hyperbilirubinemia was $7.716 \mathrm{mg} / \mathrm{dl}$ as compared to a value of $5.154 \mathrm{mg} / \mathrm{dl}$ in those who did not. The difference was significant $(\mathrm{p}=0.000)$. Using Receiver operating characteristic (ROC) curve analysis, a value of $6.4 \mathrm{mg} / \mathrm{dl}$ (first day TSB) was determined to have the best predictive ability for subsequent hyperbilirubinemia with a sensitivity of $87.5 \%$, specificity of $80.11 \%$, positive predictive value of $37.5 \%$ and a negative predictive value of $97.92 \%$.

Awasthi and Rehman [21], with the 1st day bilirubin level of $\geq 3.99 \mathrm{mg} / \mathrm{dl}$ showed that it has a sensitivity $68.6 \%$, specificity $71 \%$, positive predictive value $35 \%$ and negative predictive 
value of $96 \%$ in predicting neonatal hyperbilirubinemia. Alpay et al. [26], with the 1 st day bilirubin level of $\geq 6 \mathrm{mg} / \mathrm{dl}$ showed that it has a sensitivity $90 \%$, specificity $65.3 \%$, positive predictive value $26.3 \%$ and negative predictive value of $97.9 \%$ in predicting neonatal hyperbilirubinemia. Triasih et al. [27], with the 1st day bilirubin level of $>4.5 \mathrm{mg} / \mathrm{dl}$ showed that it has a sensitivity $90 \%$, specificity $71.9 \%$, positive predictive value $50 \%$ and negative predictive value of $96.8 \%$ in predicting neonatal hyperbilirubinemia.

The present study infers that 1st day (24hrs) bilirubin $>5.7 \mathrm{mg} / \mathrm{dl}$ can also be used as an early predictor of neonatal hyperbilirubinemia. Knowing the fact that still many deliveries are being conducted at home or health centers where laboratory investigations facilities are not available, or by the time these babies reach to higher center it may not be possible to obtain cord blood due to dried up cord. In such scenario, first day bilirubin may be used to predict significant neonatal hyperbilirubinemia with sensitivity of $90 \%$ and positive predictive value of $36 \%$. In the present study we enrolled only term neonates, hence applying this study to preterm remains a question and we need a large number trial that enrolls both term and preterm neonates. The other limitation included the long term follow up of the enrolled neonates in the study.

\section{Conclusion}

It is recommended to have cord blood bilirubin and cord blood albumin estimation of all healthy term babies delivered in an institution to prevent the dangerous consequences of neonatal hyperbilirubinemia like kernicterus. Cord serum bilirubin being more sensitive than cord serum albumin, is more effective to pick the babies who develop significant neonatal hyperbilirubinemia. Since all newborn are not delivered at hospitals, blood investigations on cord blood may not be possible when they reached to hospitals. In such cases first day bilirubin may help in prediction of significant hyperbilirubinemia. The neonatologist must understand the significance of hyperbilirubinemia and also understand the long term morbidity which kernicterus can cause. The neonatologist can take cord bilirubin can an early predictor of significant hyperbilirubinemia and these at high risk neonates need to be followed up for jaundice and aggressively treated when requirement of phototherapy is there.

\section{Disclosure}

"There are no prior publications or submissions with any overlapping information, including studies and patients."

"The manuscript has not been and will not be submitted to any other journal while it is under consideration by Journal of Maternal fetal and Neonatal medicine"

Dr. Neeraj and Dr. Mukesh wrote the first draft of the manuscript.

Dr. Deepak and Dr. Santosh helped in writing manuscript and did primary corrections in the manuscript.

Dr. Suresh and Dr. Santosh made final corrections of manuscript before submission

There was no honorarium, grant, or other form of payment given to anyone to produce the manuscript.
All the authors approved the submission of this version of the manuscript and takes full responsibility for the manuscript; None of the authors have any conflict of interest.

\section{References}

1. Olusanya BO, Ogunlesi TA, Slusher TM (2014) Why is kernicterus still a major cause of death and disability in low-income and middleincome countries? Arch Dis Child 99(12): 1117-1121.

2. Sharma D, Murki A, Murki S, Pratap T (2014) Anti-M antibodies as a cause of intrauterine fetal death and neonatal hyperbilirubinaemia. BMJ Case Rep doi: 10.1136/bcr-2014-203534.

3. Sharma D, Dannapuneni N, Murki S, Pratap T (2015) Combined Anti e and Anti C Rh Isoimmunisation and Severe Hyperbilirubinemia. Indian J Pediatr.

4. Johnson L, Bhutani VK (2011) The clinical syndrome of bilirubininduced neurologic dysfunction. Semin Perinatol 35(3): 101-113.

5. Watchko JF, Tiribelli C (2013) Bilirubin-induced neurologic damagemechanisms and management approaches. N Engl J Med 369(21): 2021-2030.

6. Gazzin S, Strazielle N, Tiribelli C, Ghersi-Egea JF (2012) Transport and metabolism at blood-brain interfaces and in neural cells: relevance to bilirubin-induced encephalopathy. Front Pharmacol 3: 89.

7. Bhutani VK, Johnson-Hamerman L (2015) The clinical syndrome of bilirubin-induced neurologic dysfunction. Semin Fetal Neonatal Med 20(1): 6-13.

8. Johnson L, Bhutani VK (2011) The clinical syndrome of bilirubininduced neurologic dysfunction. Semin Perinatol 35(3): 101-113.

9. Wusthoff CJ, Loe IM (2015) Impact of bilirubin-induced neurologic dysfunction on neurodevelopmental outcomes. Semin Fetal Neonatal Med 20(1): 52-57.

10. Le LT, Partridge JC, Tran BH, Le VT, Duong TK, et al. (2014) Care practices and traditional beliefs related to neonatal jaundice in northern Vietnam: a population-based, cross-sectional descriptive study. BMC Pediatr 14: 264.

11. Habib HS (2013) Impact of discharge timings of healthy newborns on the rates and etiology of neonatal hospital readmissions. J Coll Physicians Surg Pak 23(10): 715-719.

12. American Academy of Pediatrics Subcommittee hyperbilirubinaemia (2004) Management of hyperbilirubinaemia in the newborn infant 35 or more week of gestation. Pediatrics 114(1): 297-316.

13. Taksande A, Vilhekar K, Jain M, Zade P, Atkari S, et al. (2005) Prediction of the development of neonatal hyperbilirubinemia by increased umbilical cord blood bilirubin. Ind Medica 9(1): 5-9.

14. Farhat R, Rajab M (2011) Length of postnatal hospital stay in healthy newborns and re-hospitalization following early discharge. $\mathrm{N} \mathrm{Am} \mathrm{J}$ Med Sci 3(3): 146-151.

15. Rostami N, Mehrabi Y (2005) Identifying the newborns at risk for developing significant hyperbilirubinemia by measuring cord bilirubin levels. J Arab Neonatal Forum 2: 81-85.

16. Maisels MJ, Kring E (1998) Length of stay, Jaundice and hospital readmission. Pediatrics 101: 995-998.

17. Satrya R, Effendi SH, Gurnida DA (2009) Correlation between cord blood bilirubin level and incidence of hyperbilirubinemia in term newborns. Paediatrica Indonesiana 49(6): 349-354.

18. Sun G, Wang YL, Liang JF, Du LZ (2007) Predictive value of umbilical 
cord blood bilirubin level for subsequent neonatal jaundice. Zhonghua Er Ke Za Zhi 45: 848-852.

19. Sahu S, Abraham R, John J, Mathew AA, George AS (2011) Cord blood albumin as a predictor of neonatal jaundice. Int J Biol Med Res 2(1): 436-438.

20. Knudsen A (1989) Prediction of the development of neonatal jaundice by increased umbilical cord blood bilirubin. Acta Paediatr Scand 78: 217-221

21. Awasthi S, Rehman S (1998) Early Prediction of Neonatal Hyperbilirubinemia. Indian J Pediatr 65: 131-139.

22. Oral E, Gezer A, Cagdas A, Pakkal N (2003) Oxytocin infusion in labor: the effect different indications and the use of different diluents on neonatal bilirubin levels. Arch Gynecol Obstet 267(3): 117-120.

23. Nahar Z, Mannan SA, Dey SK, Mitra U, Selimuzzaman SM (2009) The value of umbilical cord blood bilirubin measurement in predicting the development of significant hyperbilirubinemia in healthy Newborn. Bangladesh J Child Health 33(2): 50-54.

24. Bernaldo AJN, Segre CAM (2004) Bilirubin dosage in cord blood: could it predict neonatal hyperbilirubinemia? Sao Paulo Med J 122: 99-103.

25. Randev S, Grover N (2010) predicting Neonatal Hyperbilirubinemia Using First Day Serum Bilirubin Levels. Indian J Pediatr 77(2): 147150.

26. Alpay F, Sarici SU, Tosuncuk HD, Serdar MA, Inanc N, et al. (2000) The value of First Day Bilirubin Measurement in Predicting the Development of Significant Hyperbilirubinemia in Healthy Term New borns. Pediatrics 106(2): 16.

27. Triasih R, Haksari EL, Surjono A (2003) The first 24-hour bilirubin level as a predictor of hyperbilirubinemia in healthy term newborns. Pediatrica Indonesiana 43: 5-6. 